\title{
A Study of Post-stroke Pain in a Tertiary Care Hospital in Eastern India
}

\author{
${ }^{1}$ Ramesh Bhattacharyya, ${ }^{2}$ Kartick C Ghosh, ${ }^{3}$ Sarbajit Das, ${ }^{4}$ Suman Das, ${ }^{5}$ Gourango P Mondal, ${ }^{6}$ Anup Bhattacharya
}

\begin{abstract}
Pain is a common symptom of stroke. The exact prevalence is not known due to lack of studies. Acute and chronic pain can occur after stroke. In this study, the prevalence of different types of chronic post-stroke pain and their characteristics are evaluated. Identifying the types of pain that can occur after stroke can help to prevent and manage those cases. A retrospective and prospective study were undertaken among 1060 patients of stroke. In this series, temporomandibular joint dysfunction (TMJD) 14\%, neck pain 40\% hemiplegic shoulder pain (HSP) $41.9 \%$, brachial plexus injury $1.03 \%$, heterotopic ossification (HO) of elbow $0.6 \%$. Compressive neuropathy due to poor positioning like median nerve entrapment neuropathy $41.2 \%$, ulnar nerve entrapment neuropathy $30.6 \%$, dependent edema of hand and forearm $27.9 \%$, complex regional pain syndrome type I (CRPS type 1) 12.3\%, pressure ulcer (PU) 31.03\%, deep vein thrombosis (DVT) 2.3\%, central post-stroke pain syndrome (CPSP) $11.03 \%$, and spasticity $21.03 \%$.
\end{abstract}

Keywords: Chronic pain syndromes, Morbidity, Post-stroke pain.

How to cite this article: Bhattacharyya R, Ghosh KC, Das S, Das S, Mondal GP, Bhattacharya A. A Study of Post-stroke Pain in a Tertiary Care Hospital in Eastern India. Bengal Physician Journal, 2018;5(3):32-35

\section{Source of support: Nil}

Conflict of interest: None

\section{INTRODUCTION}

A stroke is a "brain attack" a sudden interruption of blood flow to the brain that causes brain damage and loss of function. Stroke is the first leading cause of long term disability worldwide. Various chronic pain syndrome occurs after stroke. Early recognition of those pain and managing promptly can prevent further damage and improve quality of life. Pathophysiology of post-stroke chronic pain is diverse. Some of them are nociceptive and some of them are neuropathic. Both central and peripheral sensitization leads to chronicity of pain. Dyspnea,

\footnotetext{
${ }^{1,2}$ Associate Professor, ${ }^{3,4}$ Post-Doctoral Resident, ${ }^{5,6}$ Professor and Head

${ }^{1-5}$ Department of Neurology, Calcutta National Medical College, Kolkata, West Bengal, India

${ }^{6}$ Department of Neurology, Agartala Government Medical College, Agartala, Tripura, India
}

Corresponding Author: Suman Das, Post-Doctoral Resident, Department of Neurology, Calcutta National Medical College, Kolkata, West Bengal, India, e-mail: dr.sumands@gmail.com dysarthria and cognitive impairment are a hindrance for elicitation of proper history and examination findings. Some chronic pain conditions are common and easily recognized like hemiplegic shoulder pain, central post-stroke pain (CPSP), some are common but underrecognized like temporomandibular joint dysfunction (TMJD), compressive neuropathy of upper limbs, and some are rare like brachial plexus injury and heterotropic ossification.

\section{MATERIALS AND METHODS}

This study includes the stroke patients attending the neurology department, outdoor $(n=593)$ or indoor $(n=467)$ at Calcutta National Medical College, Kolkata from October 2015 to September 2017. Some of the patients treated before attending the neurology department were evaluated retrospectively ( $n=318)$ and the rest $(n=742)$ from our prospective study. Inclusion criteria were the strength of the affected organ $<4 / 5$ as measured by the Medical Research Council Scale. Stroke occurring $>3$ months before study entry, pain duration $>3$ months, pain in visual analogue scale(VAS) $>4$ for the last 7 days. Exclusion criteria were pre-stroke pain, use of $>1$ non-opioid, or opioid analgesic for pain, use of analgesics for any other acute or chronic pain, hemi-neglect, medical instability, and impaired memory. A thorough history including history of pain in any part of the body, starting from head to toe were taken keeping in mind nociceptive (aching, throbbing) neuropathic (paresthesia, dysesthesia) and mixed character of pain. Pain score was done by VAS. Clinical examinations were done to detect neurological, musculoskeletal and other abnormality. Consultation of Orthopedician, rheumatologist and physical medicine experts were taken when necessary to identify proper causes of chronic pain. Detail sensory examination was done including touch (a wisp of cotton), pain (pin), temperature (hot and cold test tubes), joint position and vibration sense (tuning fork). Cortical sense for 2 point discrimination (2 point discriminator), graphesthesia (pencil), stereognosis. For somatic pain, inspection (color change, swelling), palpation (tenderness) and autonomic changes (swelling, temperature and trophic changes) were noted. Investigations including a blood test, neuroimaging [X-ray, color Doppler, computed tomography (CT) scan and Magnetic Resonance Imaging 
(MRI)], electrophysiology (EMG, NCV) and others were performed to confirm the diagnosis.

\section{OBSERVATIONS}

Only those patients who finished the study were taken total number of patient were 1060. Among them $44.06 \%$ were male and 55.94\% $(\mathrm{n}=503)$ were female. $70 \%$ ( $n=742)$ had infarction, $21.9 \%(n=233)$ were below 40 years of age. Hypertension noted among 50.9\% $(n=540)$, hypercholesterolemia $17.3 \%(n=184)$. Tobacco smoking $16.2 \%(n=172)$ and diabetes mellitus in $56.1 \%(n=595)$ mean VAS score $6 \pm 2$.

Temporomandibular joint (TMJ) dysfunction occurred in $13.9 \%(n=148)$. Neck pain in $40 \%(n=424)$ shoulder pain $41.9 \%(n=445)$ (Table 1$)$. On imaging by MRI of shoulder joint various causes noted were rotator cuff tendinopathy $17.9 \%(\mathrm{n}=80)$. Shoulder subluxation $22.4 \%(n=100)$, Deltoid tendinopathy $9.4 \%(n=42)$ rotator cuff tear $11.9 \%$ (53) biceps tear $13 \%$ (58\%) deltoid tear $7.8 \%$ (35), labral ligamentous complex abnormalities 6.9\% $(\mathrm{n}=31)$, others $10.3 \%)(\mathrm{n}=46)$ (Table 2$)$.

Nearly $1.03 \%(n=11)$ had brachial plexus injury. Heterotopic ossification of elbow were noted in $0.6 \%$ $(\mathrm{n}=7)$, compressive neuropathy due to poor positioning involved median nerve $41.2 \%(\mathrm{n}=437)$ and ulnar nerve $30.6 \%(n=325)$.

Dependent edema of hand and forearm were elicited in $27.9 \%(n=296)$. CRPS type I developed in $12.3 \%$ $(n=130)$, pressure ulcer in $31.03 \%(n=329)$. Deep vein thrombosis $2.3 \%$ hemorrhagic stroke, $0.56 \%$ ischemic stroke. CPSP most popular form of post-stroke pain occurred in $11.03 \%(n=117)$. Spasticity developed in $21.03 \%(n=223)$. It was determined by the modified Ashworth scale.

Table 1: Pain syndrome

\begin{tabular}{lll}
\hline Causes & Number & $\begin{array}{l}\text { Percentage } \\
(\%)\end{array}$ \\
\hline $\begin{array}{l}\text { Shoulder pain } \\
\text { Median nerve compression }\end{array}$ & 445 & 41.9 \\
neuropathy & 437 & 41.2 \\
Neck pain & 424 & 40 \\
Pressure ulcer & 329 & 31.03 \\
Ulnar nerve compressive & 325 & 30.6 \\
neuropathy & & \\
Dependent edema of hand and & 296 & 27.9 \\
forearm & & \\
Spasticity & 223 & 21.03 \\
TMJ dysfunction & 148 & 13.9 \\
CRPS type 1 & 130 & 12.3 \\
Post-stroke pain syndrome & 117 & 11.03 \\
Deep vein thrombosis & 25 & 2.3 \\
Brachial plexus injury & 11 & 1.03 \\
Heterotopic ossification of elbow & 7 & 0.6 \\
\hline
\end{tabular}

\section{DISCUSSION}

Temporomandibular joint dysfunction (TMD, TMJD) is an umbrella term covering pain and dysfunction of the muscle of mastication and temporomandibular joints. TMJD reported to be strongly associated with a decrease in chewing function which is contributing to dysphagia, malnutrition and bad oral health in post-stroke hemiparesis. In this study TMJD prevalence is $13.9 \%$. Other study prevalence of TMJD is between 5 to $12 \% .{ }^{1}$ Neck pain occurs from bottom of the head to top of shoulders. It can spread to the upper back or arms. Pain comes from an unusual position of joints resulting from muscle tightness, spasticity or stiffness. For the entire adult population, the mean prevalence is $29.80 \%$. $^{2}$ There is hardly any study depicting the prevalence of neck pain in stroke. In this study, it is $40 \%$ higher than that of the population in the community. Hemiplegic shoulder pain causes considerable distress and reduced activity and can markedly hinder rehabilitation. The aetiologies are multifactorial and ideal management is prevention. Prevalence of shoulder pain in stroke population is $40 \%{ }^{3}$ Prevalence of shoulder pain among adults of age 30 to 70 years is $22.9 \%$. ${ }^{4}$ Shoulder pain starts within days to weeks of stroke onset and incidence is slightly higher in the male. Our study shows it to be $41.9 \%$. All patient ( $n=445$ ) of hemiplegic shoulder pain undergone MRI (1.5T) by $\mathrm{T}_{1}$ and $\mathrm{T}_{2}$ weighted multi-planner and multisequence magnetic imaging. All scan were reviewed by one radiologist.

Full-thickness muscle tear was detected by full thickness gap in the entire width of the muscle both coronal and sagittal image. Partial thickness tear detected by an abnormal signal in the bursal and articular surface in $\mathrm{T}_{2}$. Tendinopathies were diagnosed by thickening and abnormal signal in proton density not in heavy $\mathrm{T}_{2} \cdot$ Labral ligamentous complex abnormalities were diagnosed by a tear of anterior part of the posterior aspect of the labrum.

There are few studies performing of MRI of the shoulder joint in hemiplegic shoulder pain. Full thick-

Table 2: MRI of shoulder

\begin{tabular}{lll}
\hline Abnormalities & Number & Percentage (\%) \\
\hline $\begin{array}{l}\text { Rotator cuff } \\
\text { tendinopathy }\end{array}$ & 80 & 17.9 \\
$\begin{array}{l}\text { Shoulder } \\
\text { subluxation } \\
\begin{array}{l}\text { Deltoid } \\
\text { tendinopathy }\end{array}\end{array}$ & 100 & 22.4 \\
$\begin{array}{l}\text { Rotator cuff tear } \\
\text { Biceps tear }\end{array}$ & 53 & 9.4 \\
$\begin{array}{l}\text { Deltoid tear } \\
\text { Labral ligamentous } \\
\text { complex }\end{array}$ & 35 & 11.9 \\
abnormalities & 31 & 13 \\
Others & 46 & 7.8 \\
\hline
\end{tabular}


ness rotator cuff tear was reported to be $20.7 \%$ in the general population. ${ }^{5}$ In another study tendon and ligament lesions were $57.1 \%$ and rotator cuff tear $38.1 \%$ in hemiplegic shoulder pain cases. ${ }^{6}$

Brachial plexus injury can occur due to shoulder subluxation or improper handling of upper extremities, and they can be confirmed by electrophysiological evidence of upper trunk involvement. Kaplan et al. presented 5 cases of hemiplegic brachial plexus injury. ${ }^{7}$

Heterotopic ossification is the abnormal growth of bone in the non-skeletal tissues including muscle, tendons or other soft tissues of which the etiology is usually either traumatic or neurogenic. Neurogenic $\mathrm{HO}$ is a known but uncommon complication that occurs after cerebral or spinal insult. The occurrence of $\mathrm{HO}$ in the post-stroke patient was reported to be $0.5-1.2 \% .^{8}$ Prolong immobilization and subsequent manipulations of joints with force to preserve ROM are the key causative factors. ${ }^{9}$ In this study, heterotopic ossification of the elbow was observed in $0.6 \%(n=7)$ of the hemiplegic elbow.

Compressive neuropathy due to poor positioning of the paretic limb may cause morbidity after stroke. Nerve conduction studies confirm the diagnosis. In one study it has been shown in the paretic limb 37.5\% suffered from median nerve entrapment neuropathy at the wrist and another $37.5 \%$ from ulnar nerve neuropathy at the elbow, whereas in non-paretic side it was $25 \%$ and $18.7 \%$, respectively. ${ }^{10}$ This study showed 41.2 and $30.6 \%$ on the paretic side whereas on the non-paretic side it was 28.9 and $10 \%$ respectively. This type of variation can occur as populations and rehabilitation differs from region to region.

Swelling and edema of hand developed due to increased venous congestion related to prolonged dependency and loss of muscle pumping function in the paretic limb. Swelling can be measured by hand volumeter or measuring tape and edema by palpation. We have used the later methods. In this study, dependent edema of hand and forehand were elicited in 27.9\% $(n=296)$. In one study edema of the hand were observed in $33 \%$ of patients. ${ }^{11}$

Complex regional pain syndrome I (CRPS type I ) is a chronic pain condition characterized by prolonged and excessive pain and changes in skin color, temperature and/or swelling in the affected arm, leg, hand or foot. In post-stroke CRPS usually involves shoulder and wrist with the elbow relatively spared. It has also named as a shoulder-hand syndrome. CRPS I can be diagnosed by the Budapest clinical diagnostic criteria. ${ }^{12}$ In our study CRPS type I were elicited in $12.3 \%(n=130)$ by the Budapest criteria. In our study CRPS type I was noted to be $15 \%$ by IASP criteria. ${ }^{3}$ This disparity can be explained by the facts that IASP criteria is more sensitive but later developed. Budapest criteria are more specific.
Pressure ulcers also called pressure sores, bedsores and decubitus ulcers are of tissue damage that occurs in people who cannot turn themselves like, stroke, acutely ill or elderly immobile persons. Categories of PU can be classified as epidermis (grade I), partial thickness dermis (grade II) partial thickness subcutaneous tissue (grade III), muscle and bone (grade IV). In this study prevalence of PU were observed to be $31.09 \%(n=329)$ which is slightly higher than that of $28 \%{ }^{13}$

Deep vein thrombosis occurs when blood clots forms in any one of the deep vein of the body usually in legs. Stroke is a risk factor for developing DVT. In one study it was noted that the incidence of DVT in hemorrhagic stroke $1.9 \%$ and ischemic stroke $0.5 \% .{ }^{14}$ This study showed the incidence of DVT were $2.3 \%(n=25)$ in hemorrhagic stroke and $0.56 \%(n=6)$ in ischemic stroke. Although the incidence is slightly higher, but the ratio remains the same.

Central post-stroke pain, formerly known as thalamic pain syndrome of Dejerine and Roussy, is a central neuropathic pain occurring in patients affected by stroke. This disease affects an estimated $8 \%$ of all stroke patients and possibly more. ${ }^{15}$ CPSP usually starts within 4 to 8 weeks of stroke but can appear within 1 year. Usually, $60 \%$ of them are thalamic in origin. In this study, CPSP was observed in $11.03 \%(n=117)$ of stroke patient which is similar to the above and other studies.

Post-stroke spasticity is a common complication associated with other signs and symptoms of the upper motor neuron syndrome. It contributes to impairment and disabilities. Clinical measures of spasticity were done by the modified Ashworth scale. Prevalence of spasticity after stroke was noted to be of $27 \%$ by modified Ashworth scale. ${ }^{16}$ Our study showed it to be $21.03 \%(n=223)$.

\section{CONCLUSION}

Chronic pain syndromes are common, even following mild to moderately severe stroke. Chronic pain is associated with greater decline in physical and cognitive health making it an important medical complication of stroke. It is a source of anxiety, depression, and a hindrance to physical rehabilitation. Early identification of the type of pain and their proper management can reduce the morbidity. Consultations of different medical specialties like orthopedics, rheumatologist, psychiatrist, internist, and radiologist are often required to pinpoint the diagnosis. Both nociceptive and neuropathic pains are recognized as a complication of stroke. Their occurrence according to descending order of frequency is shoulder pain, median nerve compression neuropathy, neck pain, pressure ulcer, ulnar nerve compressive neuropathy, dependent edema of hand and forearm, spasticity, TMJ dysfunction, CRPS 
type 1 , post-stroke pain syndrome and brachial plexus injury.

\section{REFERENCES}

1. Johansson A, Unell L, Carlsson GE, et al. Gender difference in symptoms related to temporomandibular disorders in population of 50 year old subjects. J Orofacial Pain 2003;17:29-35.

2. Cole P, Cassidy JD, Caroll L. The Saskatchewan Health and Back Pain Survey. The prevalence of neck pain and related disability in Saskatchewan adults. Spine 1998;23:1689-1698.

3. Pinedo S. Complication in the hemiplegic patient in the first year after stroke. Rev Neurology 2001;32:206-209.

4. Bhawna, Multani NK, Kundu ZS. Prevalence of shoulder pain among adults in Northern India. Asian Journal of Health and Medical Research 2016;2:18-22.

5. Yamanato A, Takashisi K, Osawa T et al. Prevalence and risk factors of a rotator cuff tear in the general population. J Shoulder Elbow Surg 2010;18:116-120.

6. Zhu Y, Su B, Li N, Jin H. Pain management of hemiplegic shoulder pain post stroke in patients from Nansu, China. Neurol Regen Res 2013;8:2389-2398.

7. Kaplan PE, Meridita J, Taft G, et al. Stroke and brachial plexus injury difficult problem. Arch Phys Med Rehabil 1977:58:415-418.
8. Crawford CM, Versgese G, Mani MM, et al. Heterotropic ossification: are range of motion exercises contraindication. J Burn Care Rehabil 1986;323:7.

9. Garland DE. A clinical perspective on common forms of acquired heterotopic ossification. Clin Orthop Related Research 1991;263:13-29.

10. Remziye Hanker, Kemal Baki. Journal of clinical Neurophysiology 2012;29:96-100.

11. Boomkamp-Koppen HG, Visser-Meily JM, Post MW, et al. Post stroke hand swelling and odema. Prevalence and relationship with impairment and disability. Clin Rehabil 2005;19: 552-559.

12. Harden RN, Bruehl S, Stanton-Hicks M, et al. Proposed new diagnostic criteria for complex regional pain syndrome. Pain Medicine 2007:6:326-331.

13. Amir Y, Halfens RJ, Lohrmann C, et al. Pressure ulcer prevalence and quality of care in stroke patients in a Indonesian Hospital. Journal of Wound Care 2013;22:254-260.

14. Gregory PC, Jhemier K, Keith V. Prevalence of venous thrombosis in acute hemorrhagic and thromboembolic stroke. Am Phys Med Rehabil 2003;82:364-369.

15. Schott GD. From thalamic syndrome to central post stroke pain. J Neurol Neurosurg Psychiatry 1996;61:560-564.

16. Watkins CL, Leathley MJ, Gregson M, et al. Clinical Rehabil 2002;16(5):515-522. 